\title{
Los dolores abdominales psicógenos de la infancia: una revisión
}

\author{
RESUMEN: Revisión bibliográfica \\ exhaustiva de los dolores abdominales \\ funcionales de la infancia. Se describe \\ su expresión clínica, el diagnóstico dife- \\ rencial, la epidemiología y los enfoques \\ explicativos biopsicosocial y evolutivo- \\ psicodinámico. \\ PALABRAS CLAVE: dolor abdominal, \\ psicológico, niños, epidemiología, revi- \\ sión, modelos explicativos.
}

\begin{abstract}
SUMMARY: Thorough bibliographic review about the functional abdominal pain in childhood. It's described its clinic expression, differential diagnosis, epidemiology and the biopsychosocial and developmental-psychodinamic explanatory models.

KEY WORDS: abdominal pain, psychological, children, epidemiology, review, explanatory models
\end{abstract}

\section{Expresión clínica del dolor abdominal funcional}

El dolor abdominal es, junto a la cefalea de tensión, la modalidad de expresión somática funcional más frecuente en la edad escolar. Son algias, acompañadas a veces de síntomas de palidez, vómitos o náuseas, que el niño suele localizar de manera vaga y poco precisa alrededor de todo su abdomen, principalmente en la región periumbilical, pero también en la región epigástrica. En ocasiones, esta localización se alterna o se irradia a otro lugar. Estos niños visitan con frecuencia los servicios médicos siendo sometidos a múltiples y costosas exploraciones que, ocasionalmente, terminan en intervenciones de apéndice innecesarias. En general, los dolores pueden atribuirse a numerosos factores y, dependiendo del sentido que tengan en cada niño, tendrán sus peculiaridades específicas.

El horario y la duración del dolor es de gran variabilidad de unos casos a otros y de unos episodios a otros. A veces las coincidencias en el tiempo están ligadas a las situaciones conflictivas: hay escolares que se quejan todas las mañanas antes de ir al colegio, otros que no quieren comer y notan la tensión cuando se acerca esta hora, etc. La frecuencia de los episodios también varía considerablemente. Hay niños con crisis muy puntuales aunque severas en su intensidad y otros que se quejan de un dolor continuo de intensidad leve o moderada que persiste durante días.

La condición de dolor abdominal recurrente en la infancia viene dada por unas características que, desde que fueron enunciadas por Apley en 1975 (1), permanecen aceptadas de un modo bastante generalizado: al menos tres episodios de dolor en los últimos tres meses, lo suficientemente severos en intensidad como para interferir en las actividades normales, y en los que se haya descartado una etiología orgánica conocida.

Rev. Asoc. Esp. Neuropsiq., 2001, vol XXI, n. ${ }^{\circ}$ 80, pp. 9-54 


\section{Diagnóstico diferencial}

A pesar de que solamente un porcentaje muy bajo de los casos de dolor abdominal se debe a causas orgánicas identificadas, es preciso hacer una completa exploración médica para poder descartar una serie de cuadros y enfermedades que pueden cursar igualmente con estos dolores.

La presencia de conflictos emocionales o síntomas comportamentales es solamente indicativa, no definitiva, para descartar causas orgánicas. Hay niños con problemas psicológicos importantes que pueden tener, a su vez, problemas físicos. La etiología orgánica y emocional no son mutuamente excluyentes y, en algunos casos, están solapadas, o bien, lo emocional se apuntala sobre una alteración orgánica sosteniendo y reforzando el síntoma.

El diagnóstico diferencial ha de incluir:

1. Un estudio psicológico del niño y de su entorno. Datos del desarrollo evolutivo, acontecimientos familiares y escolares recientes y pasados, circunstancias que rodean al síntoma, historia de enfermedades, etc.

2. Una exploración física. Se precisa del diagnóstico médico que, habitualmente, incluye exámenes del recuento sanguíneo, análisis y cultivos de orina y heces, análisis de parásitos, exámenes radiológicos del bajo vientre y del tracto gastrointestinal, radiografías $\mathrm{y} / \mathrm{u}$ otras exploraciones médicas que se consideren pertinentes. En el estudio físico se deben descartar:

- Patologías del tracto gastrointestinal como: úlcera péptica, pólipos, adenitis, quistosis mesentérica, apendicitis, etc.

- Patologías neurológicas: epilepsia, tumores cerebrales, etc.

- Patologías del tracto urinario: como pielonefritis, cálculos renales, infecciones urogenitales u otras.

- Patologías hepáticas o pancreáticas: pancreatitis, fibrosis quística, etc.

- Enfermedades metabólicas: hipoglucemia, porfiria, etc.

Entre un 10 y un $33 \%$ de niños con dolor abdominal funcional son apendicectomizados (2-5). Frecuentemente tras la operación reaparecen los dolores, confirmando así su etiología funcional. Este dato tan alarmante nos hace pensar sobre la importancia de un diagnóstico diferencial preciso.

\section{Datos epidemiológicos}

La incidencia del dolor abdominal oscila entre un $8 \%$ y un $15 \%$ de los niños en edad escolar (1), (6-13). Los casos en los que puede justificarse plenamente su etiología orgánica son entre el 5 y el 10\%, según autores (1), (14-16). Al 90-95 \% restante se le atribuyen, de manera primordial, causas psicológicas en su etiología. 


\subsection{Sexo y edad}

A continuación presentamos dos de los trabajos epidemiológicos más relevantes sobre dolor abdominal en edades escolares:

Apley y Naish (6), en una muestra de 1000 niños ingleses de 3 a 15 años y clase social media baja, indican que: el 10'8\% (9'5\% en niños y $12^{\prime} 3 \%$ en niñas) tiene al menos tres episodios de dolor lo suficientemente intensos como para afectar sus actividades cotidianas, durante un período de al menos 3 meses.

En niños entre 5 y 10 años, hay una incidencia que varía entre el 10 y el $12 \%$, seguida por un progresivo descenso ( $7 \%$ a los 12 años). En niñas, la incidencia es de la misma tendencia aunque ligeramente inferior que en los niños hasta los 8 años. Después hay un fuerte incremento (hasta un $25 \%$ de las niñas de 9 años), seguido de un progresivo descenso (13\% a los 12 años).

La edad más frecuente de inicio de estos dolores se sitúa en los 5 años y medio en los dos sexos (edad que ponen en relación con el inicio de la escolaridad). También hay un importante número de niñas que empieza a quejarse a los 9 y 10 años.

Mortimer y otros (12), en un trabajo con 1083 niños entre 3 y 11 años, muestran que: el $8.4 \%$ de la muestra tiene dolor abdominal recurrente ( $8 \%$ de los niños y $9 \%$ de las niñas). El dolor alcanza su máxima prevalencia entre los 5 y los 7 años (un $10.7 \%$ de los niños y un $11.1 \%$ de las niñas). Entre 3 y 5 años se da en el $8^{\prime} 7 \%$ de los niños y el 9' 8 $\%$ de las niñas. Entre 7 y 9 años, el 7' $5 \%$ de los niños y el 7'1\% de las niñas. Entre 9 y 11 años, el 5'6\% de los niños y el 5'8\% de las niñas.

Tanto en los estudios de Mortimer y otros (12) y Apley y Naish (6), como en otras investigaciones (17-19), el porcentaje final de niños y niñas con dolor abdominal durante la escolaridad primaria es similar. Los porcentajes de ambos sexos varían en función de las edades.

\subsection{Incidencia familiar}

Independientemente de la polémica de si son los modelos patógenos de los padres los que provocan una identificación por parte del niño o si los factores de déficit constitucional familiar tendrían un peso preponderante, un ambiente familiar de quejas somáticas puede ser un factor a tomar en cuenta en las experiencias de dolor del niño $(20,21)$.

El $46 \%$ de los niños con dolor abdominal tiene algún familiar (padres o hermanos) con este síntoma, mientras que, en el grupo control, sin síntomas abdominales, sólo un $8 \%$ de los niños tiene algún familiar con dolor (6). Stone y Barbero (22), en una muestra de 102 niños entre 2 años y medio y 14 años, indican que un $28 \%$ de madres y un $20 \%$ de padres de niños con dolor abdominal tienen problemas gastrointestinales de algún tipo. Oster (7) señala que entre los niños con dolor abdominal de su muestra, el 16 $\%$ de las madres y el $17 \%$ de los padres, tienen dolor abdominal.

McGrath y otros (23) obtienen resultados contrapuestos a los anteriores. En dos grupos de 30 niños con dolor abdominal y 30 controles, con una media de edad de 11 años, los padres de niños con dolor abdominal sufren menos el síntoma que los padres de niños sin dolor abdominal ( $24 \%$ por $29 \%$ ). 
Christensen y Mortensen (24) afirman que los hijos de padres que durante su infancia tuvieron dolor abdominal no diferían en la variable dolor de los hijos de padres que no tuvieron dolor abdominal en su infancia. Sin embargo, en el momento de la investigación, se encuentra una mayor incidencia de dolor abdominal en los hijos de padres con dolor abdominal (13\%) que en los hijos de padres sin dolor abdominal (5\%).

Aunque la mayoría de los trabajos muestran que entre los hijos de padres con dolor abdominal hay más casos con dolor abdominal que entre hijos de padres que no tienen dolor abdominal, no hay una completa unanimidad.

\subsection{Otras variables sociodemográficas}

En este apartado presentamos algunos resultados obtenidos de la observación de otras variables sociodemográficas como lugar de nacimiento, nivel social, nivel educativo, etc.

Para Zuckerman y otros (11) los factores sociodemográficos sexo del niño, edad de la madre, lugar de nacimiento, nivel de educación o clase social no diferencia a los niños con o sin dolor abdominal. Alfven (25), en una muestra sueca, señala que los niños pertenecientes a clase social baja o inmigrantes, sufren dolor abdominal en una proporción casi el doble que los niños de nivel sociocultural alto (27' $5 \%$ por $\left.15^{\prime} 1 \%\right)$. En el grupo seleccionado por Faull y Nicol (10) no se observa una asociación positiva entre dolor abdominal recurrente y número de hermanos, posición del niño en la fratría o sexo del niño, pero sí encuentran relación entre niños con dolor abdominal y padres que se comunican peor entre sí. Golding y Butler (26) no observan diferencias entre el grupo con dolor abdominal y el grupo control en las variables clase social, edad de la madre, situación de pareja entre los padres o grupo étnico de pertenencia. Campo y otros (27) encuentran que los somatizadores provienen de clase social baja, de padres que no viven juntos y de etnias minoritarias.

En general no hay resultados concluyentes que muestren una relación positiva entre las variables clase social, edad de la madre, relación entre los padres, lugar geográfico de nacimiento y nivel educativo y la presencia de dolor abdominal.

\subsection{La asociación dolor abdominal-cefalea tensional}

La somatización es frecuentemente polisintomática, y los síntomas somáticos parecen agruparse en la población pediátrica (28). Hay una fuerte evidencia empírica para poder hablar de un síndrome de quejas de dolor, que se repite en ciertas edades y sexos (29).

Las quejas de dolor abdominal y cefaleas tensionales recurrentes son bastante comunes en la infancia. Esta circunstancia ha propiciado el estudio de su asociación sintomática (1), (6), (7), (10), (22), (26), (30), (31), (32), (33). Estos autores coinciden en que la agrupación de dolores abdominales y de dolores de cabeza son modalidades de expresión de problemas emocionales y en muchos casos parte de un modelo familiar de trastorno. 
Entre un 22 y un $33 \%$ de los niños con uno de estos síntomas (dolor abdominal o dolor de cabeza) tiene también el otro (9), (11), (30). Mortimer y otros (12) afirman que el $67 \%$ de los niños y el $71 \%$ de las niñas con dolor abdominal recurrente tienen o tuvieron en algún momento de su vida cefaleas recurrentes. De los niños sin dolor abdominal, el $55 \%$ de los niños y el $53 \%$ de las niñas tienen o tuvieron en algún momento de su vida cefaleas. Alfven (25) informa que el 58\% de un grupo de niños con dolor abdominal también tiene dolores de cabeza frecuentes.

Frecuentemente, ambos dolores aparecen con el inicio de la escolaridad, aunque un gran número de autores coinciden en señalar que la somatización progresa desde el algia abdominal hasta la cefalea (1), (7), (22), (24), (30).

\section{Enfoques explicativos del dolor abdominal funcional}

Las propuestas explicativas sobre el síntoma de dolor abdominal dependen del marco teórico de cada clínico. En esta revisión general de la bibliografía, nos hemos propuesto exponer dos enfoques totalmente diferentes en sus concepciones: el enfoque biopsicosocial y el enfoque evolutivo-psicodinámico.

\subsection{Enfoque biopsicosocial}

Para los autores de esta perspectiva el síntoma se desencadena cuando los factores psicosociales actúan sobre factores de predisposición biológica al dolor (9), (22), (34), (35).

Se han hecho investigaciones empíricas con métodos estandarizados de evaluación y comparaciones con grupos control para estudiar sistemáticamente las características psicológicas y del entorno social que tienen los niños vistos en las consultas pediátricas por dolor abdominal funcional. Se destacan los siguientes:

\section{a. Factores de personalidad}

Estos niños son más tímidos, tensos, ansiosos, exigentes y vulnerables a la tensión emocional que los que no tienen estos síntomas (1). Para Stone y Barbero (22) son tensos, con un nivel de tolerancia a la frustración muy bajo, sensibles, aprensivos e inseguros. Barr y Feuerstein (4), les atribuyen características de ansiedad, inhabilidad social y tendencia al perfeccionismo.

Numerosos estudios se han preocupado por demostrar que, en comparación con grupos control, los niños con dolor abdominal tienen más características depresivas (21), (36), (37), (38), (39), (40), (41), (42), (43), (49) y de ansiedad (1), (4), (21), (22), (37), (39), (41), (42), (43), (44), (45), (46), (47), (49).

También se ha señalado que los niveles de ansiedad y depresión que tienen los niños con dolor abdominal recurrente son igual de importantes en otros grupos de niños con enfermedades orgánicas (20), (23), (42), (48).

Tampoco está claro que la naturaleza de los trastornos emocionales sea diferente en niños con dolor abdominal recurrente y en niños con otros trastornos psicológicos. Los niveles de ansiedad pueden ser tan altos en unos como en otros (41), (49). 
La literatura demuestra que las investigaciones llevadas a cabo en relación a las características de personalidad (principalmente sus manifestaciones psicopatológicas de ansiedad y depresión) no diferencia los grupos con dolor abdominal recurrente de grupos de niños con enfermedades orgánicas o con otro tipo de trastornos emocionales, aunque si los diferencia respecto a grupos control.

\section{b. Factores ambientales}

Ya hemos visto previamente que un ambiente familiar con frecuentes manifestaciones de dolor puede ser, para el niño, un factor social de riesgo de somatización. La transmisión del dolor de padres a hijos puede darse por identificaciones. El niño aprende e imita un modelo de síntoma existente en algún miembro de la familia, recibiendo por ello beneficios secundarios (1), (6), (17), (20), (22), (24), (40), (45), (50). McGrath y otros (23) no llegan a estas conclusiones. Además, hay autores que indican que entre los padres de niños con dolor abdominal había más propensión a la cefalea que al propio dolor abdominal (7).

Además, se ha señalado que estos niños tienen más experiencias estresantes en los meses previos al inicio del dolor (46) y están expuestos, generalmente, a mayor tensión emocional (22), (44), (51).

Autores como Stone y Barbero (22), Crossley (52), Hughes (38), Routh y Ernst (20) Wasserman y otros (21) o Campo y otros (27) citan entre los factores desencadenantes más comunes: situaciones de tensión relacionadas con el colegio, tales como ansiedad ante los exámenes o fobias escolares, o con el ambiente familiar, tales como separaciones, entorno familiar hostil, historia de enfermedades en los padres, hechos traumáticos, psicopatología de los padres o actitudes familiares inadecuadas (exceso de preocupación por el niño y por su salud, altas expectativas). Pero, aunque los niños con dolor abdominal recurrente puedan tener un mayor número de episodios recientes de acontecimientos vitales negativos que grupos normales (9), (46), no difieren de pacientes con otras patologías (9) o de pacientes orgánicos (23), (43), (48).

Minuchin y otros (53), Sanders y otros (54) Walker y otros, (47) o Scharff (55) afirman que no está clara la asociación entre familias disfuncionales y niños con síntomas de abdominalgias.

Pensamos que las investigaciones psicosociales de este enfoque no llegan a constituir un verdadero modelo teórico explicativo sino que son una prolongación de la epidemiología descriptiva. Estos estudios han logrado resultados parciales en la búsqueda de sus objetivos. Podemos resumir las siguientes conclusiones:

- No existe una relación específica entre determinadas características de personalidad y niños con dolor abdominal.

- Los factores de aprendizaje por identificación son factores de riesgo en la aparición de una somatización, pero la modalidad de somatización no es siempre la misma en padres y niños.

- Tampoco hay una relación específica clara entre determinados acontecimientos vitales y el desencadenamiento de dolor abdominal. 


\subsection{Enfoque evolutivo-psicodinámico}

Desde esta perspectiva, se considera que existe un entrelazamiento del síntoma con los estadíos madurativos que atraviesa el niño en su evolución. Vamos a centrarnos, en primer lugar, en la psicodinámica evolutiva, donde se diferencia entre la patología abdominal entroncada en una disarmonía evolutiva y la entroncada en un contexto edípico y, seguidamente, nos ocuparemos de la diversidad de contextos psicopatológicos en los que se encuadra el dolor.

\subsubsection{Contexto evolutivo del dolor abdominal}

Los dolores abdominales funcionales se presentan en edades específicas y muestran hasta qué punto los trastornos deben relacionarse estrechamente con la maduración del funcionamiento de los diferentes órganos y con las características del desarrollo psicológico. No representan lo mismo los dolores abdominales en el cólico idiopático del primer trimestre, que los dolores del inicio de la escolaridad o los de entrada en la pubertad. Están ligados al desarrollo psicosexual del niño e influidos de manera significativa por las perturbaciones existentes en el medio ambiente.

Para Gutton y Estrabaud (56) en el abdomen se encuentra la imagen condensada de todo un pasado libidinal y agresivo. "Esta imagen se constituye a través de los procesos primarios de semejanza y contigüidad respecto a las zonas erógenas” (pág. 438).

El proceso de semejanza se expresa a partir de un doble aspecto arcaico de la imagen del cuerpo:

- Los orificios. En el abdomen, el ombligo es su expresión sustitutiva, además de ser su prototipo histórico prenatal.

- El envoltorio. Es el revestimiento cerrado muscular-cutaneo.

Alrededor de estos dos elementos se organizan las nociones fundamentales de la estructuración arcaica del cuerpo: nociones de dentro y de fuera, de penetración $y$ de expulsión y de lleno y vacío. El ombligo es el revestimento abdominal "que condensa" en su topología todo el cuerpo. Es preciso concebir esta condensación como un proceso dinámico en el cual, la división arcaica se integra en beneficio de sus equivalencias simbólicas.

Las relaciones de contigüidad se efectúan con los orificios erógenos inferiores (en los dolores abdominales bajos) y con la boca (en la topografía abdominal alta). El vientre es un lugar privilegiado donde se integran las demandas orales, los fantasmas edípicos y los mecanismos regresivos.

En la historia del vientre, la madre tiene un lugar privilegiado. El abdomen, por su contenido vacío o lleno, está directamente entroncado con dos preocupaciones biológicas maternales: la alimentación y las heces, que constituyen, a su vez, la erogeneidad de la boca y del ano. Por tanto, para la madre, esta parte del cuerpo tiene un verdadero valor simbólico de la calidad de vida de su hijo. Más tarde, el vientre es objeto de las teorías sexuales infantiles y engendra fantasmas que alcanzan los registros oral, anal y genital. De ellos surgen innumerables interrogantes e hipótesis del niño sobre la diferencia de los sexos, el contenido misterioso del vientre o sobre la procreación (56). 
La psicodinámica evolutiva del dolor abdominal puede estar asociada tanto a un contexto de disarmonía evolutiva como a un repliegue ante la angustia edípica en un contexto edípico.

\section{a. Disarmonía evolutiva ${ }^{l}$}

Los dolores abdominales, cuando se producen en un contexto de disarmonía evolutiva, son la expresión de un presente perturbado y de un pasado conflictivo. Las algias representan un sistema de interacción arcaica que indica malestar y angustia, y revela la profunda herida narcisista y el investimento corporal ambivalente (57).

El contexto psicológico es el del sadismo reprimido, dirigido originariamente hacia una madre desvalorizada y ligado a la ausencia o inadecuación de la identificación con el padre (57). Marcelli y Ajuriaguerra (58) señalan la imposibilidad tanto para el niño como para los padres (especialmente la madre) de expresar socialmente la vertiente agresiva inevitable de las relaciones.

La abdominalgia permite el desplazamiento de la agresividad hacia el cuerpo del niño, logrando invertir el sadismo encubierto en cuidados y solicitud exagerada hacia el síntoma del niño. La agresividad, que no está contrainvestida eficazmente, es introyectada por el niño de manera masoquista. Su automatismo de repetición tiene el valor de acting.

\section{b. Contexto edípico}

En la latencia podemos encontrar el dolor abdominal asociado a un repliegue ante la angustia edípica que provoca una regresión hacia los modos de relación de la organización pregenital. El fantasma de castración hay que ligarlo generalmente con un incidente desencadenante que puede ser físico (abdominal o no) o psicológico (frustración, prohibición de la masturbación). El dolor puede estar asociado (56):

- A una práctica masturbatoria. La abdominalgia aparece como un equivalente autoerótico que sustituye a la masturbación (práctica culpabilizada y reprimida).

- Al temor inconsciente a sufrir un daño en las partes genitales. En estos casos el dolor puede desplazarse hacia la parte alta del abdomen (este desplazamiento puede llegar a dar a la cabeza, en forma de cefaleas, un valor sustitutivo al abdomen). Algunas veces se asiste a un mecanismo de reaseguramiento oral hacia la integridad del vientre; el niño come o bebe algo y el dolor desaparece. Es un medio mágico para verificar el buen funcionamiento de los órganos y para llenar el vientre, que corre el riesgo de haber sido vaciado o destruído fantasmáticamente. Se trata de una defensa frente a la angustia de castración.

\subsubsection{Contexto psicopatológico del dolor abdominal}

Los dolores abdominales se caracterizan por la diversidad y el polimorfismo de su expresión. Van desde trastornos leves que acompañan a situaciones temporales de adaptación y que no tienen otros signos de patología asociada, hasta trastornos psicoafectivos deficitarios graves que desembocan en patologías psicosomáticas crónicas. 
El dolor abdominal puede ser un simple modo de expresión reactiva del niño ante un conflicto pasajero, donde la utilización de su propio cuerpo forma parte de la evolución psicoafectiva normal. Al igual que otros síntomas (por ejemplo la fobia), el dolor abdominal puede aparecer en momentos críticos de organización del yo. El carácter normalizador de estos episodios aislados queda explicado con la siguientes palabras de Benhamou y Gutton (60):

"La movilidad de las expresiones sintomáticas y el aspecto transitorio de los momentos regresivos certifican la flexibilidad necesaria para un desarrollo mental satisfactorio".

En ocasiones, una conducta de adaptación episódica resulta difícil distinguirla del conflicto internalizado utilizado para manipular al entorno o de la simulación. Es común en el periodo de latencia y se asocia frecuentemente a situaciones que crean ansiedad. El dolor está claramente relacionado con circunstancias emocionales y el niño trata de explotar las ventajas que obtiene (no ir al colegio, no comer algo que no le gusta, llamar la atención, dormir con los padres, etc.).

La repetición del síntoma resulta un inquietante criterio, indicativo de que el niño tropieza con una etapa de su evolución. Cuando los dolores se convierten en una pauta recurrente, podemos observar diferentes niveles de elaboración mental que pueden estar intrincados en el síntoma abdominal. Benhamou y Gutton (60) describen tres niveles que confieren al síntoma valores psicopatológicos distintos:

\section{1- La expresión abdominal de la angustia}

$\mathrm{Su}$ explicación parte del contexto fisiológico de la emoción. Aparece en el transcurso de acontecimientos desagradables para el niño, donde la causa psicológica se encuentra fácilmente. Se observa en niños particularmente emotivos con un pasado de dolor abdominal y con dificultad para verbalizar.

"Todo ocurre como si el dolor abdominal fuera el testigo de una brusca transformación del objeto externo en objeto psíquico sin la defensa del lenguaje y del juego" (56).

El acontecimiento provoca la hospitalización urgente en razón de la ansiedad extrema de alguno de los padres. En estos casos habría que evaluar cuidadosamente el papel del médico y de la familia y el contexto de hospitalización, que tienen importancia en el incremento de la angustia. También ha de analizarse el acontecimiento dentro el marco de la psicopatología infantil tratando de entender el significado en el inconsciente del niño.

A esta repetición de la angustia traumática Benhamou y Gutton (60), oponen los dolores abdominales de mayor elaboración psíquica que se encuentran dentro de un contexto edípico.

\section{2- La identificación}

El dolor abdominal puede resultar de una elaboración mental definida por el mecanismo psíquico de la identificación y la problemática de la castración. Uno de los 
familiares cercanos sufre o ha sufrido dolor abdominal. El síntoma del niño tiene un valor resolutivo simbólico ante el conflicto que generan determinados deseos edípicos prohibidos. Las identificaciones sintomáticas edípicas surgen al modo de secuencias de comunicación con el rival del mismo sexo o con el objeto de amor.

En estos casos se encuentran presentes los mecanismos psíquicos característicos de la conversión histérica: nivel edípico de elaboración de conflictos, complejo juego de identificaciones, riqueza del contenido de los conflictos emocionales y fantasmáticos (angustia de castración), intereses genitales marcados pero reprimidos, intrincación pulsional del síntoma expresando la hetero y autoagresividad en un rico régimen libidinal, habilidad de las conductas regresivas y sugestionabilidad hacia la aparición dolorosa y la desaparición (curación). Fácilmente se asiste a la renuncia del síntoma cuando es desenmascarado el sentido (60).

\section{3- La angustia de fragmentación}

Con menor frecuencia el síntoma abdominal aparece como la expresión de temores arcaicos de despersonalización y fragmentación. En los casos de mayor gravedad son niños con estructuras psicoafectivas mal constituidas, con dificultades en sus procesos de organización mental, vacíos de psicodinámica y que presentan el dolor abdominal como forma de vivir un cuerpo que de otro modo no podrían sentir que es suyo. En estos casos el síntoma está más próximo a funcionamientos borderline y psicóticos.

\section{Agradecimiento}

A todos los compañeros, colegas y amigos que con la lectura y comentarios de este texto han contribuido a enriquecerlo.

\footnotetext{
1 El concepto de disarmonía evolutiva, utilizado por autores como Lebovici o Ajuriaguerra, pretende señalar el desajuste que se establece entre las lineas en curso de maduración. Este concepto deriva de lo que Anna Freud (59) denomina lineas de desarrollo; ella señala que estas líneas "representan el resultado de la interacción entre el desarrollo del yo, del superyo y de sus reacciones frente a las influencias del medio, es decir, entre los procesos de maduración, adaptación y estructuración" (pág. 56), "trazan el gradual crecimiento del niño desde las actitudes dependientes, irracionales, determinadas por el ello y los objetos hacia un mayor control yoico del mundo interno y externo" (pág. 55). El efecto desorganizador de la disarmonía surge cuando el yo y el superyo no poseen la madurez suficiente para controlar las pulsiones parciales pregenitales y agresivas.
} 


\section{BIBLIOGRAFIA}

(1) Apley, J., The child with abdominal pains, Oxford: Blackwell Scientific Publications, 1975.

(2) Jess, P.; Bjerregaard, B., Brynitz, S., "Acute appendicitis: prospective trial concerning diagnostic accuracy and complications". American Journal of Surgery, 1981, 141, p. 232-234.

(3) Creed, F.H.; Lennard-Jones, J.E., “Gastrointestinal symptoms". En F. Creed y J.M. Pfeffer (Eds), Medicine and psychiatry: a practical approach, Massachusetts: Pitman, 1982.

(4) Barr, R.G.; Feuerstein, M., Recurrent abdominal pain syndrome: how appropiate are our basic assumptions?" En P.J. McGrath y P. Firestone (Eds), Pediatric and adolescent behavioral medicine, New York: Springer, 1983. p. 13-27

(5) Heafield, R.; Roe, A.M., Watkins, R., Brodribb, A.J., Brown, C., "Outcome of emergency surgical admissions for non-specific abdominal pain”, Gut, 1990, 31, A1167.

(6) Apley, J.; Naish, N., "Recurrent abdominal pains: a field of survey of 1.000 school children", Archives of Disease in Childhood, 1958, 33, 165-170.

(7) Oster, J., "Recurrent abdominal pain, headache and limb pains in children and adolescents", Pediatrics, 1972, 50, 3, 429-436.

(8) Parcel, G.S.; Nader, P.R., Meyer, M.P., “Adolescent health concerns, problems and patterns of utilization in a triethnic urban population”, Pediatrics, 1977, 60, 157-166.

(9) Hodges, K.; Kline, J.J., Barbero, G., Flaney, R., "Life events occurring in families of children with recurrent abdominal pain", Journal of Psychosomatic Research, 1984, 28, 3, 185-188.

(10) Faull, C.; Nicol, A.R., "Abdominal pain in six-year-olds. An epidemiological study in a new town", Journal of Child Psychology and Psychiatry, 1986, 27, 251-260.

(11) Zuckerman, B.; Stevenson, J., Biley, V., "Stomachaches and headaches in a community sample of preschool children", Pediatrics, 1987, 79(5), 677-682.

(12) Mortimer, M.J.; Kay, J., Jaron, A., "Clinical epidemiology of childhood abdominal migraine in an urban general practice", Developmental Medicine and Child Neurology, 1993, 35, 243-248.

(13) Hyams, J.S., "Abdominal pain and irritable bowel syndrome in adolescents: a community based study", Journal of Pediatrics, 1996, 129, 220-226.

(14) Stickler, G.B.; Murphy, D.B., "Recurrent abdominal pain", American Journal of Disease in Childhood, 1979, 133, 486-489.

(15) Rappaport, L., "Recurrent abdominal pain: theories and pragmatics", Pediatrician, 1989, $16,78-84$.

(16) Boyle, J.T., "Recurrent abdominal pain: an update", Pediatr. Rev., 1997, 18, 310-321.

(17) Dodge, J.A., "Recurrent abdominal pain in children", British Medical Journal, 1976, 1, 385-387.

(18) Maloney, M., "Diagnosing hysterical conversion reactions in children", Journal of Pediatrics, 1980, 97, 1016-1020.

(19) Goodyer, I.M., "Hysterical conversion reactions in childhood", Journal of Child Psychology and Psychiatry, 1981, 22, 179-188.

(20) Routh, D.K.; Ernst, A.R., "Somatization disorder in relatives of children and adolescents with functional abdominal pain", Journal of Pediatric Psychology, 1984, 9, 427-437. 
(21) Wasserman, A.L.; Whittington, P.F., Rivara, F.P., "Psychogenic basis for abdominal pain in children and adolescents", Journal of the American Academy of Child and Adolescent Psychiatry, 1988, 27, 179-184.

(22) Stone, R.T.; Barbero, G.J., "Recurrent abdominal pain in childhood", Pediatrics, 1970, 75 (5), 732-738.

(23) Mcgrath, P.J.; Goodman, J.T., Firestone, P., Shipman, R., Peters, S., "Recurrent abdominal pain: a psychogenic disorder?" Archives of Disease in Childhood, 1983, 58, 888-890.

(24) Chistensen, M.J.; Mortensen, O., "Long-term prognosis in children with recurrent abdominal pain", Archives of Disease in Childhood, 1975, 50, 110-114.

(25) Alfven, G., "The covariation of common psychosomatic symptoms among children from socioeconomically differing residential areas. An epidemiological study", Acta Paediatrica, 1993, 82, 484-487.

(26) Golding, J.; Butler, N.R., "Headaches and stomachaches". En J. Golding y N.R. Butler (Eds), From birth to Five, Oxford: Pergamon Press, 1986. (cap. 9)

(27) Campo, J.V.; Jansen-Mcwilliams, L., Comer, D.M., Kellher, K.J., "Somatization in pediatric primary care: association with psychopathology, functional impairment and use of services", Journal of the American Academy of Child and Adolescent Psychiatry, 1999, 38 (9), 1093-1101.

(28) Garber, J.; Walker, L.S., Zeman, J., "Somatization symptoms in a community sample of children and adolescents: further validation of the Children's Somatization Inventory", Journal of Consulting and Clinical Psychology, 1991, 3 (4), 588-595.

(29) Achenbach, T.M.; Conners, C.K., Quay, H.C., Verhulst, F.C., Howell, C.T., "Replication of empirically derived syndromes as a basis for taxonomy of child/adolescent psychopathology", Journal of Abnormal Child Psychology, 1989, 17, 299-323.

(30) Cobo, C., Paidopsiquiatría dinámica, Madrid: Servicio Científico "Roche”, 1983.

(31) Shapiro, E.; Rosenfeld, A.A., The somatizing child, New York: Springer-Verlag, 1987.

(32) Kristjansdottir, G., "Prevalence of pain combinations and overall pain: a study of headache, stomach pain and back pain among schoolchildren", Scand. J. Soc. Med, 1997, 25, 58-63.

(33) Egger, H.L.; Costello, E.J., Erkanli, A., Angold, A., "Somatic complaints and psychopathology in children and adolescents: stomach aches, musculoeskeletal pains, and headaches", Journal of the American Academy of Child and Adolescent Psychiatry, 1999, 38 (7), 852-860.

(34) Engström, I., "Mental health and psychological functioning in children and adolescents with inflammatory bowel disease: a comparision with children having other chronic illnesses and with healthy children", Journal of Child Psychology and Psychiatry, 1992, 33 (3), 563-582.

(35) Woodbury, M.M., "Recurrent abdominal pain in child patients seen at a pediatric gastroenterology clinic. Observations of 50 children and their families", Psychosomatics, 1993, 34 (6), p. 485-493.

(36) Kashani, J.; Barbero, G., Bolander, F., "Depression in hospitalized pediatric patients", Journal of the American Academy of Child Psychiatry, 1981, 20, 123-134.

(37) Astrada, C.A.; Licamele, W.J., Walsh, T.L., "Recurrent abdominal pain in children and associated DSM-III diagnosis", American Journal of Psychiatry, 1981, 138, 687-688.

(38) Hughes, M.C., "Recurrent abdominal pain and childhood depression: clinical observation of 23 children and their families", American Journal of Orthopsychiatry, 1984, 54, 146-155. 
(39) Ernst, A.R.; Routh, D.K., Harper, D.C., "Abdominal pain in children and symptoms of somatization disorder", Journal of Pediatric Psychology, 1984, 9, 77-85.

(40) Eisendrath, S.J.; Way, L.W., Ostroff, J.W., Johanson, C.A., "Identification of psychogenic abdominal pain", Psychosomatics, 1986, 27 (10), 705-712.

(41) Garber, J.; Zeman, J., Walker, L.S., "Recurrent abdominal pain in children: psychiatric diagnosis and parental pathology", Journal of the American Academy of Child and Adolescent Psychiatry, 1990, 29, 648-656.

(42) Walker, L.S.; Greene, J.W., "Children with recurrent abdominal pain and their parents: more somatic complaints, anxiety and depression than other families?" Journal of Pediatric Psychology, 1989, 14, 231-243.

(43) Walker, L.S.; Greene, J.W., "Negative life events and symptom resolution in pediatric abdominal pain patients", Journal of Pediatric Psychology, 1991, 16, 341-360.

(44) Mckeith, R.; O'neill, D., "Recurrent abdominal pain in children”, The Lancet, 1951, $18,278-282$.

(45) Hughes, M.C.; Zimin, R., "Children with psychogenic abdominal pain and their families", Clinical Pediatrics, 1978, 17 (7), 569-573.

(46) Robinson, J.O.; Alvarez, J.H., Dodge, J.A., "Life events and family history in children with recurrent abdominal pain", Journal of Psychosomatic Research, 1990, 34 (2), 171-181.

(47) Walker, L.S.; Garber, J., Greene, J.W., "Psychosocial correlates of recurrent childhood pain: a comparision of pediatric patients with recurrent abdominal pain, organic illness, and psychiatric disorders", Journal of Abnormal Psychology, 1993, 102 (2), 248-258.

(48) Raymer, D.; Weininger, O., Hamilton, J.R., "Psychological problems in children with abdominal pain", The Lancet, 1984, 25, 439-440.

(49) Hodges, K.; Kline, J.J., Barbero, G., Woodruff, C., "Anxiety in children with recurrent abdominal pain and their parents", Psychosomatics, 1985, 26 (11), 859-866.

(50) Farquhar, H.G., “Abdominal migraine in children”, British Medical Journal, 1956, 1, 1082-1085.

(51) Green, M., "Diagnosis and treatment: psychogenic, recurrent, abdominal pain", Pediatrics, 1967, 40 (1), 84-89.

(52) Crossley, R.B., "Hospital admissions for abdominal pain in childhood", Journal of the Royal Society of Medicine, 1982, 75, 772-776.

(53) Minuchin, S.; Baker, L., Rosman, B.L., Liebman, R., Milman, L., Todd, T.C., “A conceptual model of psychosomatic illness in children”, Arch. Gen. Psychiatry, 1975, 32, 1031-1038.

(54) Sanders, M.R.; Morrison, M., Rebgetz, M., Bar, W., Dadds, M., Shepard, R., "Behavioral treatment of childhood recurrent abdominal pain, children's psychological characteristics, and family functioning", Behavior Change, 1990, 7, 16-24.

(55) Scharff, L., "Recurrent abdominal pain in children: a review of psychological factors and treatment", Clinical Psychology Review, 1997, 17 (2), 145-166.

(56) Gutton, P.; Estrabaud, M., "Les douleurs abdominales psychogenes", Revue de Neuropsychiatrie Infantile, 1975, 23 (7), 425-447: p. 431

(57) Sirol, F., Los dolores abdominales recurrentes de la última infancia y de la adolescencia. En S. Lebovici, R. Diatkine y M. Soule (Eds), Traitê de psychiatrie de l'enfant et de l'adolescent. Paris: Presses Universitaires de France. (Traducción castellano: Tratado de Psiquiatría del Niño y del Adolescente. Vol. 4. Madrid: Biblioteca Nueva, 1990). 
(58) Marcelli, D.; Ajuriaguerra, J., Psicopatología del niño. Barcelona: Masson. $3^{\text {a }}$ ed., 1996.

(59) FREUD, A., Normality and pathology in childhood. New York: International University Press. (Traducción castellano: Normalidad y patología en la niñez. Buenos Aires: Paidos. $5^{\text {a }}$ reimpresión, 1984. Orig. 1965).

(60) BENHAMOU, H.; GUTTON, P.H., "Les douleurs abdominales psychogènes de l'enfant", Perpectives Psychiatriques, 1983, 2 (91), 131-134; p. 133

* Psicólogo Clínico. Centro de Salud Mental Sur. Hospital Virgen del Rocío. Sevilla. **Psiquiatra. Servicio de Psiquiatría. Hospital General Universitario de Murcia ***Psiquiatra. Práctica Privada. Madrid

Correspondencia: Dr. Juan Otero

c/Andrés Mellado, $81,2^{\circ} \mathrm{C}$, esc. izda. 28003 , Madrid.

Email: juanotero01@mixmail.com

Fecha de recepción: 17-VII-01 